\title{
Carcinoma medular de tiroides: Experiencia de 20 años*
}

\author{
Drs. ENRIQUE LANZARINI S. ${ }^{1}$, ANDRÉS MARAMBIO G. ${ }^{1}$, JOSÉ AMAT V. ${ }^{1}$, FRANCISCO RODRÍGUEZ M. ${ }^{1}$, \\ PATRICIO GAC E. ${ }^{1}$, PATRICIO CABANÉ T. ${ }^{1}$, RODRIGO LOEHNERT T. ${ }^{1}$, JUAN PABLO MARAMBIO G. ${ }^{1}$
}

1 Departamento de Cirugía Hospital Clínico Universidad de Chile, Santiago, Chile.

\begin{abstract}
Medullary thyroid carcinoma: 20 years experience

Background: Medullary thyroid carcinoma (MTC) is a rare malignant tumor that arise from C cells. Surgical treatment and its results are controversial, so we decided to study it. Aim: To describe clinically MTC, treatment and outcomes in the long term. Material and Method: We retrospectively reviewed medical records of patients with MTC operated in our hospital between the years 1987 and 2007. We analyzed the clinical characteristics, treatment, morbidity and long-term follow up. Results: There were 24 patients operated with a mean age of $46.1 \pm 16.6$ years. The main form of presentation was painless increased cervical volume $(56.2 \%)$. In $15 \%$ this pathology was part of a MEN $2 \mathrm{~b}$. All of them have had a total thyroidectomy, which was extended in $50 \%$ of cases. The $35.2 \%$ were multifocal, $29.4 \%$ bilateral and $62.5 \%$ had metastatic lymph node involvement. Five patients remained higher calcitonin levels in the postoperative period and 9 patients recurred clinically on average 4.5 years after surgery. The presence of persistent disease was significantly associated with hereditary MTC $(p=0.0088)$ and the clinical recurrence was significantly determined by the presence of not expanded total thyroidectomy $(\mathrm{p}=0.0196)$. The probability of surviving more than 19 years was $66.6 \%(95 \% \mathrm{CI}=0.24$ to 0.89$)$. Conclusions: The $\mathrm{MTC}$ is a rare tumour and treatment of choice is surgery. The persistent disease is associated with hereditary MTC form, and the clinical recurrence is associated with not expanded total thyroidectomy. We recommend total thyroidectomy with central voiding and radical modified jugular dissection.
\end{abstract}

Key words: Medullary thyroid carcinoma, calcitonin, MEN.

\section{Resumen}

Antecedentes: El carcinoma medular de tiroides (CMT) es un tumor maligno poco frecuente, originado a partir de las células C. Su tratamiento quirúrgico y resultados son controvertidos, por lo que hemos decidido estudiarlo. Objetivo: Describir clínicamente el CMT, tratamiento y resultados a largo plazo. Material y método: Se revisaron retrospectivamente las fichas clínicas de pacientes con CMT operados en nuestro hospital entre 1987 y el 2007. Se analizaron las características clínicas, tratamiento, morbilidad y seguimiento a largo plazo. Resultados: Se operaron 24 pacientes, cuya edad media fue 46,1 $\pm 16,6$ años. La principal forma de presentación fue aumento de volumen cervical (56,2\%). Un 15\% formaba parte de una NEM 2b. A

*Recibido el 21 de Septiembre de 2009 y aceptado para publicación el 15 de Octubre de 2009.

Correspondencia: Dr. Andrés Marambio G.

Santos Dumont 999, Santiago, Chile

Código Postal: 8380456. Fax: 56-2-7370844

E-mail: amarambio@med.uchile.cl 
todos se les realizó una tiroidectomía total, ampliada en el 50\% de los casos. El 35,2\% eran multifocales, el $29,4 \%$ bilaterales y el $62,5 \%$ tenía metástasis ganglionar. Cinco pacientes mantuvieron niveles de calcitonina elevados en el postoperatorio y nueve pacientes recurrieron clínicamente, en promedio, a los 4,5 años. La enfermedad persistente se asoció significativamente con CMT hereditario $(\mathrm{p}=0,0088)$ y la recurrencia clínica a tiroidectomía total no ampliada $(\mathrm{p}=0,0196)$. La probabilidad de sobrevivir más de 19 años fue $66,6 \%$ (IC $95 \%=0,24$ a 0,89). Conclusiones: EL CMT es un tumor raro cuyo tratamiento de elección es la cirugía. La persistencia de enfermedad se asocia con la forma hereditaria, y la recurrencia clínica con la tiroidectomía total no ampliada, lo que nos hace recomendar una tiroidectomía total asociada a vaciamiento central y disección yugular radical modificada.

Palabras clave: Carcinoma medular de tiroides, calcitonina, NEM.

\section{Introducción}

El CMT corresponde a una neoplasia de las células $\mathrm{C}$, las cuales tienen por función regular el metabolismo del calcio. Tiene una baja frecuencia alcanzando un $5 \%$ de todos los cánceres tiroideos ${ }^{1,2}$. Suele presentarse en forma esporádica $(84 \%)^{3}$ o hereditaria $(16 \%)$, esta última en el contexto de un CMT familiar o de una neoplasia endocrina múltiple tipo 2 (NEM 2), la cual se asocia a distintas mutaciones del proto-oncogen $\mathrm{RET}^{4}$. Tiene la característica de secretar calcitonina, marcador útil para el estadiaje, enfermedad residual y seguimiento de los pacientes a largo plazo. Es una neoplasia relativamente agresiva, ya que a pesar de su lenta progresión, al momento del diagnóstico un $60-80 \%$ tiene metástasis linfáticas ${ }^{5-9}$ lo que dificulta una terapia totalmente curativa, ya que se ha visto que después de la primera cirugía más del $50 \%$ de los pacientes mantienen niveles de calcitonina elevada ${ }^{10,11}$. Los pacientes con la forma hereditaria tienden a ser más jóvenes y a presentar una enfermedad de curso más agresivo, con un cáncer que suele ser multifocal y bilateral $^{12}$. La terapia de primera línea es la cirugía, la cual hasta ahora ha sido controversial y no bien estandarizada ya que dependerá de varios factores tales como la precocidad del diagnóstico, clínica, estadío, marcadores, etc.

El objetivo principal de este trabajo es describir nuestra experiencia analizando en forma retrospectiva la forma de presentación, hallazgos clínicos y bioquímicos, tipo de cirugía y evolución postoperatoria. Es de nuestro interés poder comparar las diferencias clínicas existente entre los distintos subtipos de CMT y su evolución a largo plazo, así como estudiar los factores asociados a una mayor tasa de enfermedad persistente y recidiva.

\section{Material y Método}

El presente estudio fue diseñado como retrospectivo y descriptivo de los casos de CMT operados en los últimos 20 años por el equipo de cirugía endocri- na del Hospital Clínico de la Universidad de Chile (HCUCH). Se recopilaron las fichas y se procedió a recolectar los antecedentes médicos personales y familiares, presentación clínica, exámenes bioquímicos y de imágenes, cirugía realizada, resultado de la biopsia, tratamientos adicionales, seguimiento clínico, de laboratorio y de imágenes, reintervenciones y sobrevida hasta el año 2007. Para esto último se realizó seguimiento telefónico y en el registro civil. Dentro de los registros se encontraban pacientes operados en el HCUCH por primera vez, como también pacientes que se habían operado previamente en otros hospitales y se reoperaron por enfermedad persistente o recidivas en el nuestro. Se consideró enfermedad persistente al alza mantenida de la calcitonina después de la cirugía o dentro de los primeros 6 meses posteriores a ésta, mientras que recidiva fue considerado como el alza en la calcitonina a partir de los 6 meses posterior a la cirugía. Los datos obtenidos fueron analizados con el programa Stata 8.1. Las pruebas estadísticas utilizadas fueron el test $\mathrm{T}$ de Student, el test de Mann Whitney, la prueba de Chi cuadrado o el test exacto de Fisher si correspondía, análisis de regresión lineal simple y logística y el método de Kaplan-Meier, considerando significativo un valor $\mathrm{p}$ menor o igual que 0,05 .

\section{Resultados}

Durante el período de estudio se operaron 24 pacientes. Cuatro fueron excluidos del análisis por no encontrarse su ficha clínica o estar incompleta. Dentro de los 20 pacientes finalmente analizados, 17 habían sido intervenidos por primera vez en nuestro hospital, mientras que 3 fueron intervenidos por recidivas. Ocho eran hombres y doce mujeres (relación 1:1,5). El promedio de edad al diagnóstico fue $46,1 \pm 16,6$ años (rango 10-68, mediana 48,5) y habitualmente esta patología se presentó como un aumento de volumen cervical indoloro $(56,2 \%)$ y en menor frecuencia, dentro del seguimiento de un nódulo tiroideo $(31,2 \%)$, o como un hallazgo durante el control de otras patologías $(6,2 \%)$. Un caso se asoció 
a una enfermedad de Basedow Graves. El tiempo de evolución previo al diagnóstico fue de 1,9 $\pm 2,6$ años en promedio (rango 1 mes a 10 años) y no hubo diferencias significativas en el tiempo de evolución si la presentación era aumento de volumen cervical o nódulo tiroideo $(\mathrm{p}=0,13)$. La mayoría de los pacientes eran eutiroideos al momento del diagnóstico (55\%), dos tenían hipotiroidismo, uno hipotiroidismo subclínico y un paciente era hipertiroideo. En tres pacientes $(15 \%)$ esta patología formaba parte de una NEM 2b. En ellos el promedio de edad al diagnóstico (23,6 $\pm 11,8$ años) fue significativamente menor $(p=0,0037)$. De los pacientes con NEM $2 b$, uno tenía una enfermedad de Hirshprung y los otros dos feocromocitomas asociados. Las características clínicas de los pacientes se pueden apreciar en la Tabla 1.

La forma ecográfica más frecuente de presentación fue como nódulo tiroideo $(69,2 \%)$, sólido o sólido-quístico, hipoecoico y de bordes irregulares asociado al depósito de microcalcificaciones. En 8 pacientes se realizó punción con aguja fina sugiriendo el diagnóstico en 4 de ellos $(50 \%)$, mientras que en el resto el diagnóstico fue realizado en el intraoperatorio. Sólo 3 pacientes contaban en su ficha clínica con medición de calcitonina plasmática previo a la cirugía, con un promedio de 178,8 pg/ml (rango 16,4-291). A todos se les realizó una tiroidectomía total, la cual fue ampliada en el 50\% de los casos, ya sea al grupo central solamente ( 5 pacientes) o asociado a una disección yugular uni o bilateral en 6 y 2 pacientes respectivamente. En un paciente se realizó una disección yugular radical, mientras que en el resto la disección yugular fue radical modificada. Se encontró asociación estadísticamente significativa entre los casos de tiroidectomía total no ampliada y la aparición posterior de recidiva clínica $(p=0,0196)$, siendo este antecedente una variable independiente importante para la predicción de la recidiva en este estudio $(p=0,045)$. Además se realizó una cirugía adicional correspondiente a una adrenalectomía bilateral en un paciente con una NEM.

En la biopsia definitiva el tamaño tumoral estaba disponible para el $75 \%$ de los pacientes, alcanzando un diámetro máximo promedio de $3,15 \mathrm{~cm}$ (rango 0,8-12). Sólo un paciente tenía un tumor menor a $1 \mathrm{~cm}$. de diámetro. El 35,2\% era multicéntrico y el $29,4 \%$ bilateral. Todos los pacientes con NEM presentaron multifocalidad y bilateralidad, diferenciándose significativamente de los pacientes con CMT esporádico, que presentaron multifocalidad en el $17 \%(p=0,01)$ y bilateralidad en el $11,7 \%$ $(\mathrm{p}=0,0058)$. El 40\% presentó compromiso capsular y sólo en el 8,3\% de las biopsias está descrito compromiso tumoral vascular. Dentro de los pacientes sometidos a tiroidectomía total ampliada el 62,5\% presentó compromiso metastásico linfático en la biopsia, con un número promedio de 9,4 linfonodos comprometidos (rango 1-22). El compromiso capsular se asoció significativamente a la presencia de metástasis linfáticas $(p=0,028)$. En un paciente está descrito un cáncer folicular concomitante. Dos pacientes recibieron tratamientos complementarios posteriores a la cirugía con radioterapia y yodo radioactivo respectivamente. Ningún paciente presentaba metástasis a distancia al momento del diagnóstico. Las características histológicas de los pacientes se pueden apreciar en la Tabla 1.

Tabla 1. Características clínicas e histológicas de los pacientes

\begin{tabular}{|c|c|}
\hline Variable & $\mathbf{n}$ \\
\hline Número de pacientes & 20 \\
\hline Edad promedio (años) & $46,1 \pm 16,6$ \\
\hline Género (Hombre/Mujer) & $8 / 12$ \\
\hline \multicolumn{2}{|l|}{ Presentación clínica*: } \\
\hline Aumento de volumen cervical & 9 \\
\hline Nódulo & 5 \\
\hline Hallazgo & 1 \\
\hline Otros & 1 \\
\hline Tiempo de evol. promedio (años) & $1,9 \pm 2,6$ \\
\hline Diagnóstico preoperatorio & 4 \\
\hline Diagnóstico postoperatorio & 16 \\
\hline \multicolumn{2}{|l|}{ Tipo de CMT: } \\
\hline Esporádico & 17 \\
\hline Familiar & 0 \\
\hline NEM 2a & 0 \\
\hline NEM $2 b$ & 3 \\
\hline \multicolumn{2}{|l|}{ Cirugía: } \\
\hline $\mathrm{TT}$ & 10 \\
\hline TTA & 10 \\
\hline \multicolumn{2}{|l|}{ Tratamiento adyuvante: } \\
\hline RT & 1 \\
\hline QMT & 0 \\
\hline $\mathrm{Y}^{131}$ & 1 \\
\hline \multicolumn{2}{|l|}{ Características histológicas: } \\
\hline Tamaño tumoral promedio (cm.) & 3,5 \\
\hline Multifocalidad & 6 \\
\hline Bilateralidad & 5 \\
\hline Compromiso capsular* & 6 \\
\hline Metástasis linfática** & 5 \\
\hline
\end{tabular}


Durante el seguimiento se contaba con los valores de calcitonina plasmática post operatoria de 10 pacientes, de los cuales $5(50 \%)$ mantuvieron enfermedad persistente con un promedio de 1905 $\mathrm{pg} / \mathrm{ml}$ (rango 144-4.782). Entre estos pacientes se encontraban todos los con NEM. Finalmente nueve pacientes $(45 \%)$ recidivaron clínicamente, en promedio a los 4,5 años (mediana 2, rango 1-15) desde la cirugía, presentando un aumento de volumen cervical en el 100\% de los casos. Entre estos pacientes se incluyen a todos los con enfermedad persistente. En 8 de estos pacientes se realizaron nuevas intervenciones, consistentes en vaciamientos ganglionares o resección de recurrencias, y al menos la mitad debió ser sometido a 2 o más reintervenciones similares. Entre los pacientes que recidivaron clínicamente, en tres $(15 \%)$ se pesquisaron metástasis a distancia (dos pacientes con adenopatías mediastínicas y un paciente con metástasis pulmonares) además de la recidiva locoregional. Tres pacientes recibieron tratamientos complementarios a la cirugía durante el seguimiento con yodo radioactivo (1 paciente), radioterapia (2 pacientes) o quimioterapia (1 paciente).

El tiempo medio de seguimiento de este estudio fue de 6,6 años (mediana 4, rango 1 mes-19 años) $y$ al término de este 3 pacientes (15\%) habían fallecido, con un promedio de sobrevida de 6,6 años (rango 3-13). Ninguno de los fallecidos presentaba una NEM y uno sólo de ellos presentaba metástasis a distancia (mediastino). La presencia de recidiva clínica se asoció significativamente al fallecimiento posterior de estos pacientes $(p=0,001)$. En esta serie no se encontraron reportes de hipoparatiroidismo permanente o lesión de nervio laríngeo recurrente en forma iatrogénica. La evolución clínica de los pacientes se resume en la Tabla 2.

El análisis estadístico final evidenció que la presencia de enfermedad persistente después de la primera cirugía estuvo asociada significativamente al tipo de CMT hereditario asociado a NEM $2 b$ $(\mathrm{p}=0,0088)$ y a una serie de variables propias de este tipo de cáncer -menor edad de los pacientes $(\mathrm{p}=0,0077)$, multifocalidad $(\mathrm{p}=0,0001)$ y bilateralidad $(\mathrm{p}=0,005)$-. Mediante un análisis de regresión logística se pudo estimar que la edad del paciente $\left(\beta_{1}=0,12 ; 95 \%\right.$ IC $=0,01$ a 0,$24 ; \mathrm{Z}=$ $2,22 ; p=0,026 ; Y=-4,08+0,12 X)$ y la presencia de bilateralidad $\left(\beta_{1}=3,68 ; 95 \%\right.$ IC $=0,68$ a 6,$69 ; Z=$ $2,41 ; \mathrm{p}=0,016 ; \mathrm{Y}=-1,38+3,68 \mathrm{X})$ en la biopsia son variables independientes importantes para predecir la presencia de enfermedad persistente luego de la primera cirugía, con un Odds ratio de 1,13 (95\% IC $=1,01-1,27)$ para la edad y un Odds ratio de 40 $(95 \%$ IC $=1,98-807,09)$ para la bilateralidad. Con la información disponible de esta serie no se identificaron otros factores, aparte de la tiroidectomía total
Tabla 2. Evolución clínica de los pacientes

\begin{tabular}{lc}
\hline Variable & n de pacientes \\
\hline Número de pacientes & 20 \\
Enfermedad persistente* & 5 \\
Recidiva Clínica & 9 \\
Tiempo de recidiva promedio (años) & 4,5 \\
Metástasis a distancia & 3 \\
Cirugía & 8 \\
Tratamiento adyuvante: & \\
$\quad$ RT & 2 \\
QMT & 1 \\
$\quad$ Y131 & 1 \\
Vivo/muerto & $17 / 13$ \\
Sobrevida promedio (años) & 6,6 \\
Seguimiento promedio (años) & 6,6 \\
\hline
\end{tabular}

*sobre un total de 10 pacientes.

Tabla 3. Variables asociadas a enfermedad persistente y recidiva clínica

\begin{tabular}{ll}
\hline Variable & p \\
\hline Menor edad & 0,0077 \\
CMT hereditario (NEM 2b) & 0,0088 \\
Tiroidectomía Total no ampliada* & 0,0196 \\
Multifocalidad & 0,0001 \\
Bilateralidad & 0,005 \\
\hline
\end{tabular}

*única variable asociada a recidiva clínica

no ampliada, que sean significativos para predecir la recidiva clínica en los pacientes operados por CMT (Tabla 3).

\section{Discusión}

El CMT es un cáncer de baja frecuencia y curso agresivo localmente, con un pronóstico intermedio entre los cánceres diferenciados y el anaplástico. Sin embargo, se ha visto que estos pacientes logran tasas de sobrevida elevadas a largo plazo ${ }^{13}$, entre un 50 a $85 \%$ a 15 años de seguimiento según Van Heerden ${ }^{11}$. En nuestra serie la probabilidad de sobrevivir más de 19 años fue de $66,6 \%(95 \%$ IC $=0,24$ a 0,89$)$ como se observa en la Figura 1. Así vemos que la mayoría de nuestros pacientes se mantienen vivos al finalizar nuestro período de estudio.

También vemos que los pacientes tratados en nuestro hospital en estos últimos 20 años son pocos, 
estimando 1 caso al año en promedio, sin embargo, llama la atención la mayor frecuencia que hemos observado durante estos últimos años (Figura 2). Debido a esto no nos es posible sacar conclusiones a más largo plazo debido al poco seguimiento que presentan estos últimos pacientes (seguimiento promedio 6,6 años para todo el grupo), asociado a ser una neoplasia de baja frecuencia.

Varios estudios han demostrado que el CMT presenta compromiso locoregional en un alto porcentaje al diagnóstico ${ }^{6-8}$ lo que constituye uno de los principales problemas que enfrentan estos pacientes, permitiendo el desarrollo de enfermedad persistente y/o recidiva. Hemos observado que al momento de la primera cirugía un $62,5 \%$ de nuestros pacientes sometidos a tiroidectomía total ampliada presentan metástasis linfáticas, porcentaje que ciertamente podría ser mayor al considerar a todo el grupo. Esto nos lleva a pensar en dos puntos: primero, en la necesidad de un diagnóstico precoz, aún su principal forma de presentación es masa cervical y a la mayoría se le realiza el diagnóstico en el intraoperatorio. Es necesario sospechar y pesquisar antes a estos pacientes para realizar el estudio adecuado y mejorar el pronóstico ${ }^{14,15}$. En esta serie sólo cuatro pacientes tenían el diagnóstico antes de la cirugía y sólo uno tenía un tumor menor a $1 \mathrm{~cm}$, lo que indica también que se operan en forma tardía. Hay que pensar que el CMT metastásico responde mal a la radioterapia y quimioterapia por lo que lo adecuado es el screening en personas en riesgo y cirugía temprana. Al respecto se ha propuesto con anterioridad la medición de calcitonina plasmática a todos los pacientes con patología nodular del tiroides ${ }^{16}$.

Como segundo punto, se necesita un enfoque quirúrgico agresivo, ya que por lo comentado el CMT se asocia a una alta tasa de enfermedad persistente y recidiva ${ }^{9}$. En nuestra serie la mitad de los pacientes presentó enfermedad persistente luego de la primera cirugía y un porcentaje similar $(45 \%)$ recidivó en

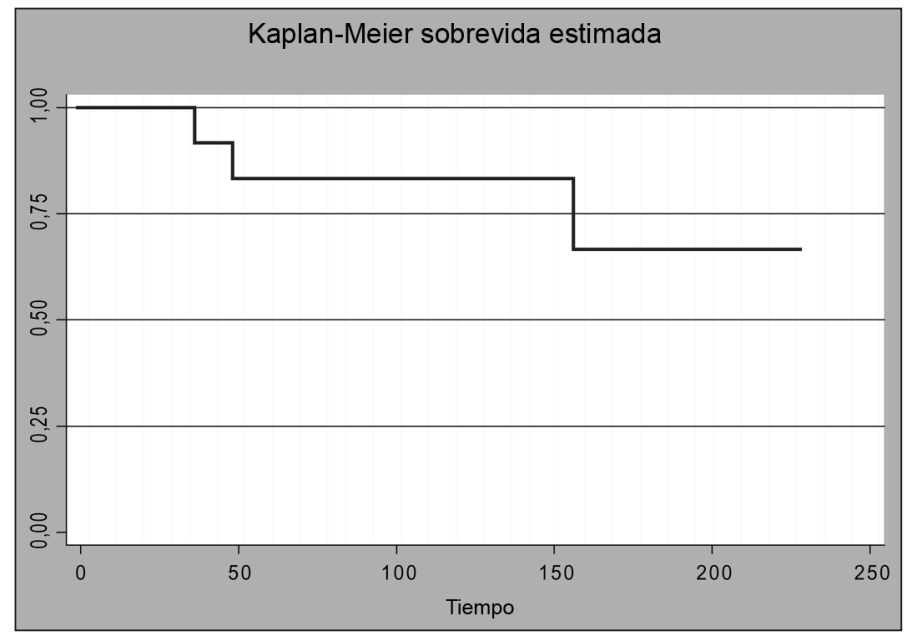

250
Figura 1. Probabilidad de sobrevida en cohorte de pacientes (20) con CMT operados en el HCUCH (tiempo en meses).

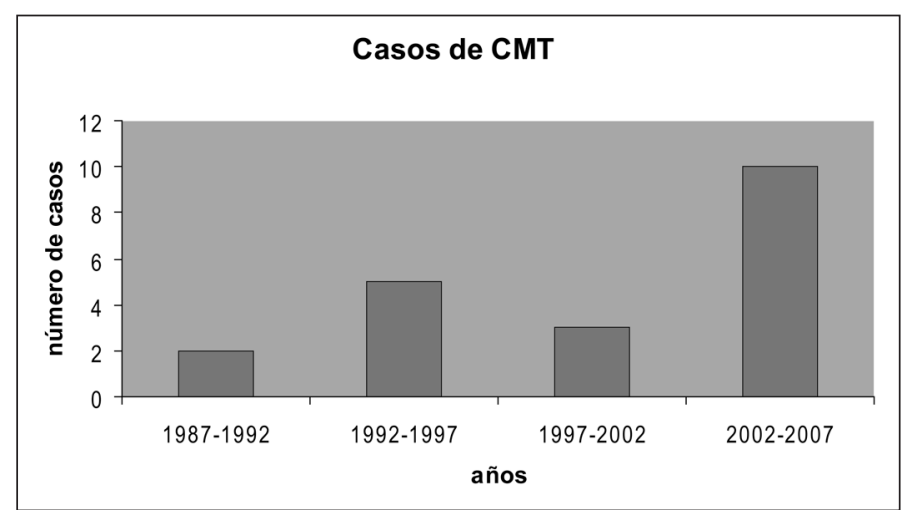

Rev. Chilena de Cirugía. Vol 62 - No 1, Febrero 2010; pág. 15-21
Figura 2. Evolución en el tiempo de frecuencia de pacientes CMT. 
el corto plazo (4,5 años en promedio), requiriendo nuevas intervenciones. Esto último se vio influido por la extensión de la tiroidectomía, evidenciándose mayor recidiva en los pacientes sometidos a tiroidectomía total no ampliada $(\mathrm{p}=0,0196)$. Hay que tener en cuenta que la cura bioquímica después de la reoperación cervical es infrecuente ${ }^{5,8,9,15,17}$, por lo que la primera cirugía es clave. Para esto es necesario tener en cuenta algunas variables que indiquen mayor posibilidad de resultado adverso. En nuestro análisis la probabilidad de enfermedad persistente estuvo asociada al CMT hereditario (NEM 2b), de reconocida mayor agresividad que su contraparte y a una serie de variables propias de este tipo, como la menor edad de los pacientes y la presencia de bilateralidad y multifocalidad. Otras variables importantes que se han identificado corresponden a los niveles de calcitonina (mientras más altos mayor la probabilidad de recidiva) ${ }^{18}$ y el estadío ${ }^{14,19-22}$. Una variable importante que encontramos fue el compromiso capsular, el cual se asoció a la presencia de metástasis linfáticas $(p=0,028)$ y de esta forma influyendo sobre un resultado adverso luego de la cirugía ${ }^{23}$.

Así podemos ver que un gran desafío es tratar adecuadamente a los pacientes con CMT hereditario, principalmente dentro del contexto de una NEM. Dentro de estas últimas la NEM $2 b$ es en general la menos frecuente, sin embargo, en esta serie sólo se presentaron casos de este tipo. A pesar de que la mayoría de las NEM $2 b$ se producen por mutaciones espontáneas nuevas en el proto-oncogen RET, no existiendo de esta forma historia familiar ${ }^{24}$, hoy se recomienda realizar a todos el estudio genético buscando mutaciones de este gen ${ }^{25}$ y así lograr un tratamiento precoz y un mejor resultado ${ }^{21}$. En nuestro caso ningún paciente contaba con estudio genético. Tampoco hay reportes de pacientes con mediciones de antígeno carcinoembrionario (otro producto de las células $\mathrm{C}$ ), que se suele usar en forma complementaria a la calcitonina, ni tampoco hay reportes de medición de calcitonina estimulada (test clínico más sensible para la medición de enfermedad persistente o recidiva). Debemos mencionar además que tenemos poca experiencia en el uso de radioterapia y quimioterapia en esta patología, las cuales son de segunda y tercera línea. También hay que decir que el yodo radioactivo no tiene ningún rol en el tratamiento actual de esta patología debido que las células $\mathrm{C}$ no captan yodo, aunque en algún momento se usó.

Podemos concluir que el CMT es poco frecuente pero agresivo localmente, asociado a altas tasas de enfermedad persistente y recidiva, aunque los pacientes viven por largos períodos. Los pacientes con CMT hereditario, específicamente dentro del contexto de una NEM $2 b$, corresponden a un grupo especial en el cual la patología se torna más agresiva con tasas más altas de enfermedad residual. Al enfrentar a un paciente con sospecha de cáncer de tiroides se debe pensar en CMT y realizar el estudio adecuado intentando realizar el diagnóstico en forma preoperatoria de forma de planificar el tratamiento adecuado, correspondiente a una tiroidectomía total ampliada, asociada a vaciamiento central y disección yugular radical modificada, idealmente bilateral ${ }^{7,17}$.

\section{Referencias}

1. Hundahl SA, Fleming ID, Fremgen AM, Menck HR. A National Cancer Data Base Report on 53,856 cases of Thyroid Carcinoma Treated in the U.S. 1985-1995. Cancer 1998; 83: 2638-2648.

2. Wells SA, Franz C. Medullary carcinoma of the thyroid gland. World J Surg 2000; 24: 952-956.

3. Alsanea O, Clark OH. Familial thyroid cancer. Curr Opin Oncol 2001; 13: 44-51.

4. Bugalho MJ, Domingues R, Rosa Santos J, Catarino AL, Sobrinho L. Mutation analysis of the RET protooncogen and early thyroidectomy: results of a Portuguese cancer centre. Surgery 2007; 141: 90-95.

5. Dottorini ME, Assi A, Sironi M, Sangalli G, Spreafico G, Colombo L. Multivariate analysis of patients with medullary thyroid carcinoma. Prognostic significance and impact on treatment of clinical and pathologic variables. Cancer 1996; 77: 1556-1565.

6. Moley JF, DeBenedetti MK. Patterns of Nodal Metastases in Palpable Medullary Thyroid Carcinoma. Recommendations for Extent of Node Dissection. Ann Surg 1999; 229: 880-888.

7. Scollo C, Baudin E, Travagli JP, Caillou B, Bellon N, Leboulleux $\mathrm{S}$ et al. Rationale for Central and Bilateral Lymph Node Dissection in Sporadic and Hereditary Medullary Thyroid Cancer. J Clin Endocrinol Metab 2003; 88: 2070-2075.

8. Fleming JB, Lee JE, Bouvet M, Schultz PN, Sherman SI, Sellin RV et al. Surgical Strategy for the Treatment of Medullary Thyroid Carcinoma. Ann Surg 1999; 230: 697-707.

9. Moley JF, DeBenedetti MK, Dilley WG, Tisell LE, Wells SA. Surgical management of patients with persistent or recurrent medullary thyroid cancer. Journal of Internal Medicine 1998; 243: 521-526.

10. Gimm O, Ukkat J, Dralle H. Determinative factors of biochemical cure after primary and reoperative surgery for sporadic medullary thyroid carcinoma. World J Surg 1998; 22: 562-567.

11. Van Heerden J, Grant CS, Gharib H, Hay ID, Ilstrup DM. Long-term Course of Patients with Persistent Hypercalcitoninemia After Apparent Curative Primary Surgery for Medullary Thyroid carcinoma. Ann Surg 1990; 212: 395-400. 
12. Kaserer K, Scheuba C, Neuhold N, Weinhäusel A, Haas OA, Vierhapper H et al. Sporadic Versus Familial Medullary Thyroid Microcarcinoma. A Histopathologic Study of 50 Consecutive Patients. Am J Surg Pathol 2001; 25: 1245-1251.

13. Clark JR, Fridman TR, Odell MJ, Brierley J, Walfish PG, Freeman JL. Prognostic Variables and Calcitonin in Medullary Thyroid Cancer. Laryngoscope 2005; 115 : 1445-1450.

14. Modigliani E, Cohen R, Campos JM, Conte-Devolx B, Maes B, Boneu A et al. Prognostic factors for survival and for biochemical cure in medullary thyroid carcinoma: results in 899 patients. Clinical Endocrinology 1998; 48: 265-273.

15. Weber T, Schilling T, Frank-Raue K, Colombo-Benkmann M, Hinz U, Ziegler R et al. Impact of modified radical neck dissection on biochemical cure in medullary thyroid carcinomas. Surgery 2001; 130: 1044-1049.

16. Pacini F, Fontanelli M, Fugazzola L, Elisei R, Romei C, Di Coscio G et al. Routine measurement of serum calcitonin in nodular thyroid disease allows the preoperative diagnosis of unsuspected sporadic medullary thyroid carcinoma. J Clin Endocrinol Metab 1994; 78: 826-829.

17. Kebebew E, Kikuchi S, Duh QY, Clark OH. Long-term Results of Reoperation and Localizing Studies in Patients With Persistent or Recurrent Medullary Thyroid Cancer. Arch Surg 2000; 135: 895-901.

18. Yen TWF, Shapiro SE, Gagel RF, Sherman SI, Lee JE,
Evans DB. Medullary thyroid carcinoma: Results of a standardized surgical approach in a contemporary series of 80 consecutive patients. Surgery 2003; 134: 890-901.

19. Chow SM, Chan JKC, Tiu SC, Choi KL, Tang DLC, Law SCK. Medullary thyroid carcinoma in Hong Kong Chinese patients. Hong Kong Med J 2005; 11: 251-258.

20. Bergholm U, Bergström R, Ekbom A. Long Term Follow-Up of Patients with Medullary Carcinoma of the Thyroid. Cancer 1997; 79: 132-138.

21. Kebebew E, Ituarte PHG, Siperstein AE, Duh QY, Clark $\mathrm{OH}$. Medullary Thyroid Carcinoma. Clinical Characteristics, Treatment, Prognostic Factors, and a Comparison of Staging Systems. Cancer 2000; 88: 1139-1148.

22. Gilliland FD, Hunt WC, Morris DM, Key CR. Prognostic Factors for Thyroid Carcinoma. A Population-Based Study of 15,698 Cases from the Surveillance, Epidemiology and End Results (SEER) Program 1973-1991. Cancer 1997; 79: 564-573.

23. Gülben K, Berberoğlu U, Boyabatli M. Prognostic Factors for Sporadic Medullary Thyroid Carcinoma. World J Surg 2006; 30: 84-90.

24. Hubner RA, Houlston RS. Molecular advances in medullary thyroid cancer diagnostics. Clinica Chimica Acta 2006; 370: 2-8.

25. Brandi ML, Gagel RF, Angeli A, Bilezikian JP, BeckPeccoz P, Bordi C et al. Guidelines for diagnosis and therapy of NEM type 1 and type 2. J Clin Endocrinol Metab 2001; 86: 5658-5671. 